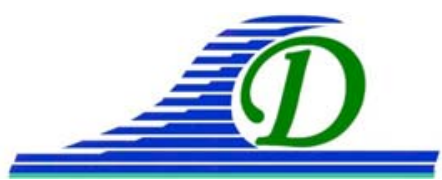

XIII İmes Journées Nationales Génie Côtier - Génie Civil

Dunkerque, 2-4 juillet 2014

DOI:10.5150/jngcgc.2014.014 @ Editions Paralia CFL

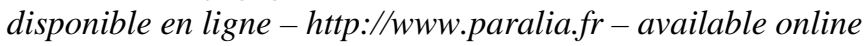

\title{
Modélisation par la méthode SPH de l'impact de la houle sur une paroi verticale.
}

\section{Xuezhou LU ${ }^{1}$, Jean-Marc CHERFILS ${ }^{2}$, Grégory PINON ${ }^{1}$, Elie RIVOALEN ${ }^{1,2}$, Jérôme BROSSARD ${ }^{1}$}

\section{Laboratoire Ondes et Milieux Complexes, LOMC UMR 6294 CNRS-Université du} Havre, 53 rue de Prony, 76058 Le Havre, France.

xuezhou.lu@doct.univ-lehavre.fr

2. Laboratoire d'Optimisation et Fiabilité en Mécanique des Structures, EA 3828, INSA de Rouen, Avenue de l'Université, BP 08, 76801 Saint- Etienne-du-Rouvray, France. elie.rivoalen@insa-rouen.fr

\section{Résumé :}

Un outil numérique, de type SPH (Smoothed Particle Hydrodynamics), a été développé dans le but de simuler l'interaction hydrodynamique entre la houle et des ouvrages portuaires ou côtiers, dotés de systèmes de récupération d'énergie marine.

Nous nous intéressons plus particulièrement à la prédiction des pics de pression survenant lors de l'impact de vagues sur un ouvrage, afin de calculer les efforts auxquels la structure sera soumise. L'impact d'un soliton sur un mur vertical, adossé à une plage inclinée, a donc été simulé dans un canal à houle numérique, montrant ainsi la capacité du code à traiter ce type de problème. En faisant varier les paramètres d'entrée du batteur, nous avons retrouvé les différentes formes d'impacts mis en évidence par les expériences : aerated impact, flip through, air pocket et slosh impact.

Afin d'étudier le lien entre la forme de l'interface et la dynamique de l'impact, un cas académique de "jet triangulaire" a été étudié de manière similaire à l'étude de (MOKRANI et al., 2012). Plusieurs variantes de la méthode SPH (Rusanov-SPH, Delta$\mathrm{SPH}, \ldots)$ ont été testées en termes de stabilité et précision. Cela a conduit à une nette amélioration du calcul de la pression sur la paroi et de la trajectoire du jet tout en préservant le caractère lagrangien de la méthode. D'autres variantes de la méthode SPH, basées sur les schémas type ALE (Arbitraire Lagrange Euler) et les solveurs de Riemann permettent d'obtenir de bons résultats (GUILCHER et al., 2010, RAFIEE et al., 2013), moyennant des temps de calculs supérieurs. Les résultats obtenus ici sont comparés avec ceux d'une autre méthode numérique, de type VOF, ainsi qu'avec un modèle analytique.

Mots-clés : Énergie marine, Hydrodynamique, Simulations numérique, Impact de vagues, Smoothed Particle Hydrodynamics. 


\section{Introduction}

Notre étude s'inscrit dans le cadre du projet national EMACOP (Energies MArines, COtières et Portuaires) qui est centré sur les ouvrages multifonctionnels faiblement immergés dotés d’un dispositif de récupération d'énergie de la houle côtière. Le dispositif sur lequel nous travaillons est un système à colonne d'eau oscillante. Il s'agit d'étudier l'impact de vagues sur l'ouvrage dans un but de dimensionnement et survivabilité de la structure. C'est sur ce point que nous focalisons nos travaux de modélisation. L'outil numérique JOSEPHINE (CHERFILS et al., 2012) que nous utilisons est un code de type SPH. C'est une méthode lagrangienne, sans maillage, bien adaptée au suivi des mouvements d'interface. C'est une méthode désormais éprouvée pour simuler des écoulements instationnaires à surface libre en grandes déformations.

\section{Description du modèle}

\subsection{Schéma d'interpolation}

Le schéma d'interpolation de la méthode SPH est basé sur la convolution des variables avec une fonction de noyau. Cette fonction est généralement à support compact et elle tend vers une mesure de Dirac lorsque le rayon de ce domaine tend vers 0 . Nous pouvons donc déterminer une approximation de la valeur d'une fonction $f$ quelconque définie sur un domaine $\Omega$ en un point de coordonnées $\vec{x}$ :

$$
f(\vec{x})=\int_{\Omega} f(\vec{y}) \eta_{\dot{o}}^{0}(\vec{y}-\vec{x}) \mathrm{dy}
$$

où : $\eta_{\dot{o}}^{0}(\vec{x})$ est la fonction de noyau. Nous avons choisi la fonction gaussienne tronquée (COLAGROSSI \& LANDRINI, 2003).

Dans la méthode SPH, le volume fluide est discrétisé par un ensemble de particules. A chacune de ces particules sont attribuées des grandeurs physiques. Cette notion de volume permet une pondération dans l'approximation discrète de l'équation (1) :

$$
f\left(\vec{x}_{i}\right)=\sum_{j} f\left(\vec{x}_{j}\right) \eta_{\dot{o}}^{0}\left(\vec{x}_{j}-\vec{x}_{i}\right) V_{j}
$$

En dérivant l'équation (2) :

$$
\nabla f\left(\vec{x}_{i}\right)=\sum_{j} f\left(\vec{x}_{j}\right) \nabla \eta_{\dot{o}}^{0}\left(\vec{x}_{j}-\vec{x}_{i}\right) V_{j}
$$

où : $V_{j}$ représente le volume de la $j^{\text {ème }}$ particule.

\subsection{Application à la résolution des équations d’Euler}

Ce schéma d'interpolation présenté peut être appliqué à la résolution des équations aux dérivées partielles. Pour un fluide faiblement compressible et non-visqueux, ce sont les équations d'Euler en description lagrangienne qui sont résolues : 


$$
\left\{\begin{array}{l}
\frac{D x}{D t}=u \\
\frac{D u}{D t}=-\frac{\nabla P}{\rho}+g \\
\frac{D \rho}{D t}=-\rho \nabla \cdot u \\
P=\frac{\rho_{0} c_{0}^{2}}{7}\left(\left(\frac{\rho}{\rho_{0}}\right)^{7}-1\right.
\end{array} \Rightarrow\left\{\begin{array}{l}
\frac{D x_{i}}{D t}=u_{i} \\
\frac{D u_{i}}{D t}=-\frac{1}{\rho_{i}} \sum_{j}\left(P_{j}+P_{i}\right) \nabla \eta_{\varepsilon}^{0}\left(x_{i}-x_{j}\right) V_{j}+g \\
\frac{D \rho_{i}}{D t}=-\rho_{i} \sum_{j}(u_{j}-u_{i}+\underbrace{n_{i j} c_{i j}\left(\rho_{i}-\rho_{j}-\Delta \rho_{i j}\right)}_{\text {Flux Rusanov }}) \cdot \nabla \eta_{\varepsilon}^{0}\left(x_{i}-x_{j}\right) V_{j} \\
P_{i}=\frac{\rho_{0} c_{0}^{2}}{7}\left(\left(\frac{\rho_{i}}{\rho_{0}}\right)^{7}-1\right.
\end{array}\right)\right.
$$

où $c_{0}$ est la vitesse du son, $P$ la pression, $g$ la gravité, $x$ le vecteur position, $u$ le vecteur vitesse, $\rho$ la masse volumique et $V$ le volume des particules.

Dans l'équation de continuité, un flux Rusanov (FERRARI et al., 2009) est ajouté pour stabiliser le schéma.

\section{Applications sur des cas d'impact de la houle sur les ouvrages}

L'objectif de ce travail est d'étudier l'impact d'une vague sur la structure. Nous avons d'abord modélisé numériquement le mouvement du batteur pour générer un soliton dans un canal à houle en 2D. Ensuite, après avoir validé la capacité du code à générer et propager une onde solitaire, nous avons ajouté une plage inclinée dans le modèle précédent pour provoquer le déferlement des solitons sur la plage ou leur impact sur la paroi.

\subsection{Génération et propagation d'une onde solitaire}

Le soliton est généré par un batteur-piston situé à l'extrémité gauche du canal. Il se propage sur le fond plat et rebondit après avoir rencontré le mur vertical situé à l'extrémité droite lorsqu'il n’y a pas de plage. La taille du canal à houle et la hauteur d'eau sont fixées. La longueur du canal est $L_{x}=5 \mathrm{~m}$, la hauteur du mur à son extrémité droite est $L_{y}=1 \mathrm{~m}$ et la hauteur d'eau $h=0,1 \mathrm{~m}$ (voir figure 1). La masse volumique est $\rho=1000 \mathrm{~kg} / \mathrm{m}^{3}$ et la gravité est $g=9.81 \mathrm{~m} / \mathrm{s}^{2}$.

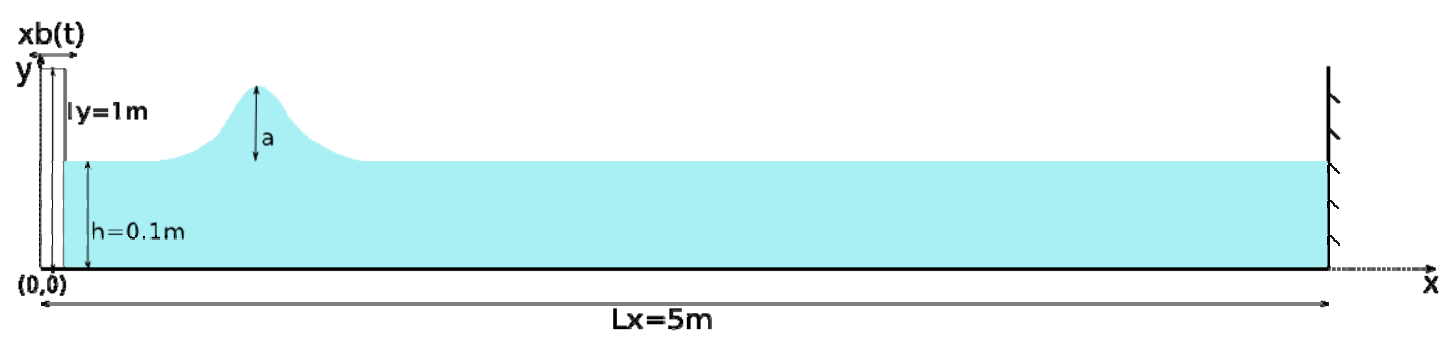

Figure 1. Vue schématique du canal à houle. 
Sur la figure $1, x_{b}(t)$ représente la loi de déplacement imposée au batteur-piston, proposée par (GORING, 1978), elle-même basée sur la théorie de (BOUSSINESQ, 1872). Cette loi permet de générer un soliton. La forme analytique du mouvement du batteur est la suivante :

$x_{b}(t)=\frac{a}{k h}\left[\tanh \left(k\left(c t-x_{b}(t)\right)+\tanh k \lambda\right)\right]$

où $c=\sqrt{g(h+a)}$ est l'approximation au premier ordre de la célérité du soliton, $k=\sqrt{\frac{3 a}{4 h^{3}}}$ et $\lambda=\frac{3.8 h}{k}$ sont deux paramètres. Il s'agit d'une équation implicite par rapport à $x_{b}(t)$, résolue numériquement par la méthode de Newton.

Différentes simulations ont été réalisées en faisant varier le rapport entre l'amplitude de l'onde et la hauteur d'eau ainsi que la résolution du maillage. Nous obtenons une bonne convergence des résultats, établie en observant l'effet de la variation du pas de temps et de la résolution spatiale sur les profils de surface libre et sur la pression maximale sur la paroi.

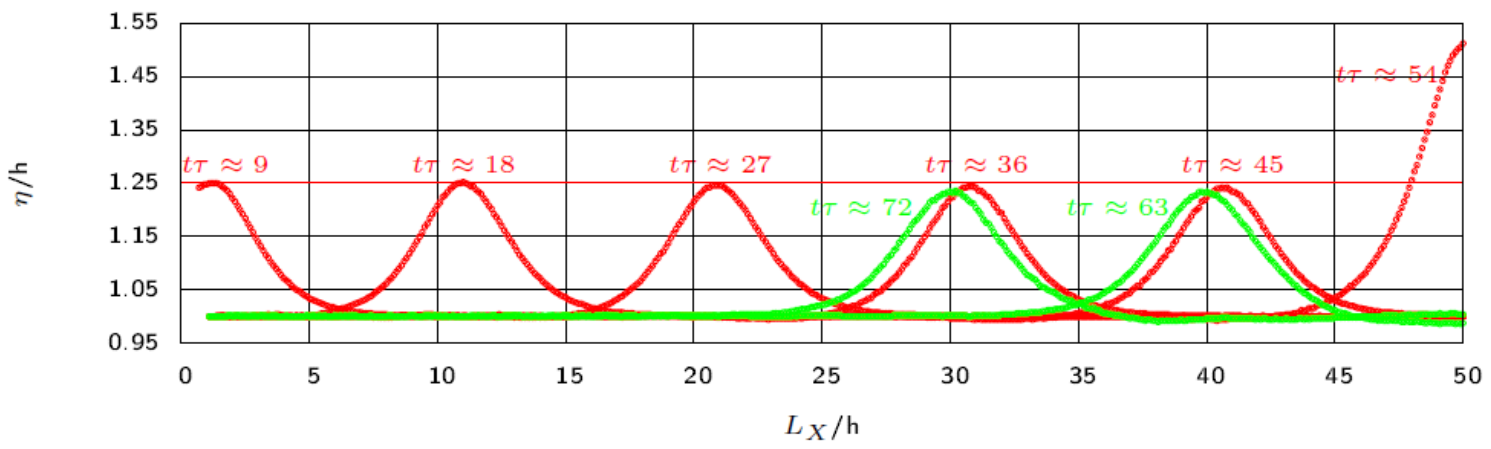

Figure 2. Progression du soliton dans le canal numérique, évolution de la surface libre.

Plusieurs positions instantanées de la surface libre du soliton pour $a / h=0,25$ sont représentées sur la figure 2, le temps $t$ est adimensionné par $\tau=\sqrt{g / h}$. Les courbes en vert représentent les instants après le rebond. Différents solitons d'amplitude croissante ont été simulés pour étudier l'évolution de la pression et de la hauteur d'eau sur le mur en fonction du rapport $a / h$.

\subsection{Déferlement d'un soliton et impact sur un mur vertical}

Dans la partie précédente, nous avons correctement simulé l'évolution d'un soliton sur un canal à fond plat. Maintenant, nous nous concentrons sur son déferlement et son impact sur une paroi verticale. Comme évoqué précédemment, nous avons ajouté une plage de pente 1:15 dans le canal (figure 3). La loi de batteur est identique à celle du cas précédent. 


\section{XIII ${ }^{\text {èmes }}$ Journées Nationales Génie Côtier - Génie Civil \\ Dunkerque, 2-4 juillet 2014}

Selon la position du point de déferlement, les impacts sont classifiés comme (HOFLAND et al. 2011) :

- "slosh impact" proche du cas du ballotement, où l'onde devrait produire une vague déferlante en aval du mur.

- "air pocket impact" ou impact à poche d'air, qui correspond au cas où l'onde déferle sur le mur, créant une poche d'aire entre l'eau et la paroi.

- "aerated impact" ou impact aéré, qui se produit quand l'onde a déferlé en amont du mur produisant un mélange air-eau avant impact sur la paroi.

- "flip through impact" qui est le cas limite et extrême entre la poche d'air et l'impact aéré, où tout l'air contenu dans la cavité est chassé au moment où la vague déferle.

Ces quatre types d'impact ont été observés au laboratoire de l'École Supérieure d'Ingénieurs de Marseille (KIMMOUN et al., 2008).

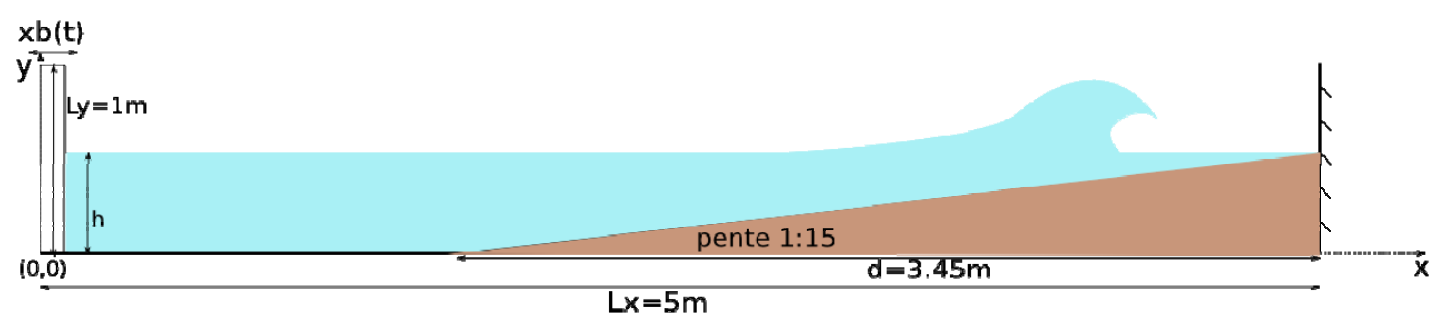

Figure 3. Vue schématique du canal à houle avec plage inclinée.

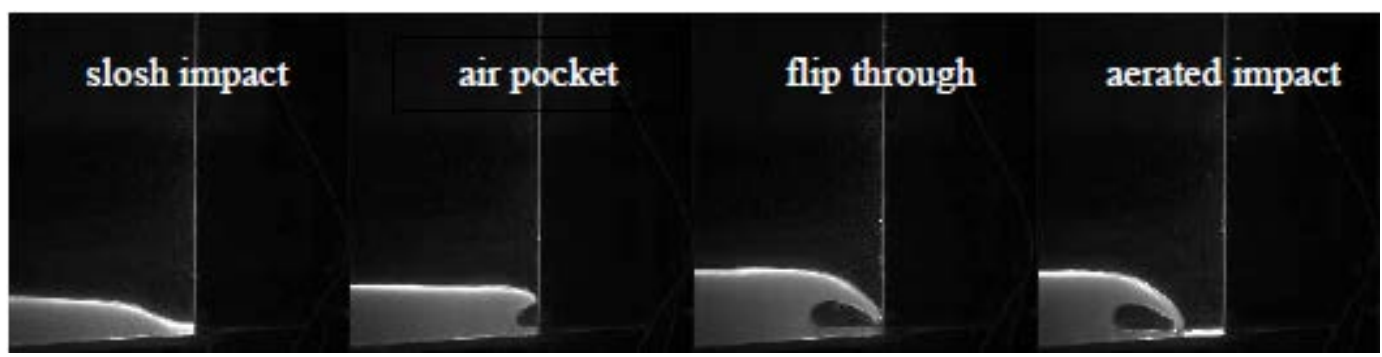

Figure 4 Différents types d'impacts obtenus par expériences.

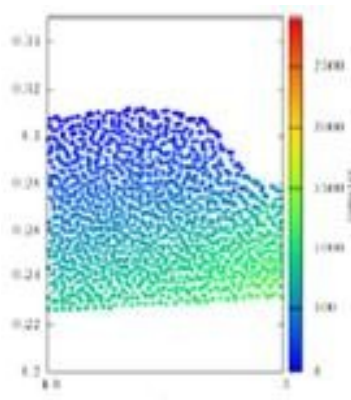

slosh impact

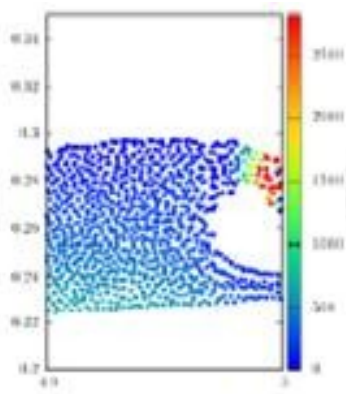

air pocket

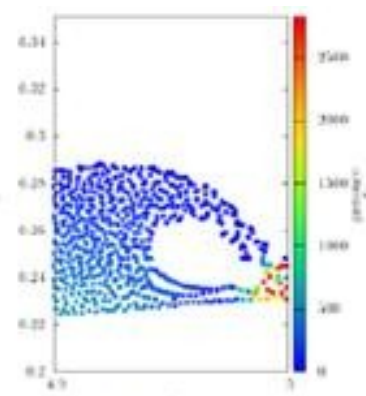

flip through

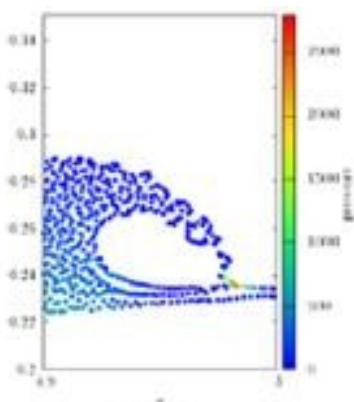

aerated impact

Figure 5 Différents types d'impacts obtenus par nos simulations. 

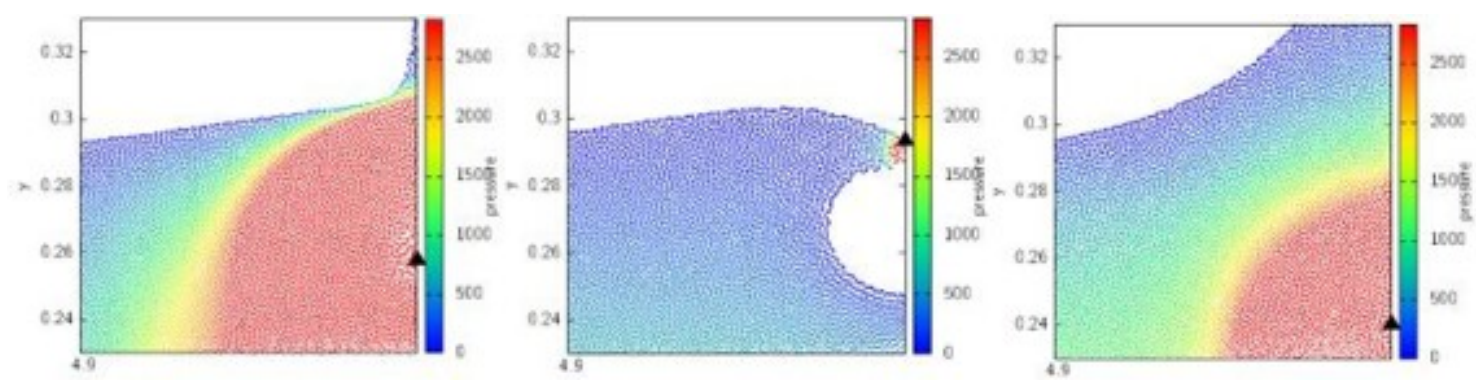

Figure 6. De gauche à droite, 1er pic à $t=4,585 \mathrm{~s}$, 2nd pic à $t=4,600 \mathrm{~s}$ et 3ème pic à $t=4.660 \mathrm{~s}$.

Nous avons bien retrouvé les différents types d’impact mis en évidence par KIMMOUN et al. (2008) en faisant varier la hauteur d'eau ou l'amplitude initiale.

Ensuite, nous nous concentrons sur le type "flip through". L'incrustation dans la Figure 7 montre la zone d'étude des grandeurs physiques : pression, force, hauteur d'eau.

Sur cette figure 7, trois pics apparaissent, ils correspondent à trois étapes successives de l'impact, représentés sur la figure 6. Les triangles noirs y représentent leurs positions. Le premier pic apparaît au moment où la crête du soliton impacte le mur. Le second pic apparaît lorsque la vague s'effondre en produisant une zone de forte pression. Le troisième pic apparaît lorsque la hauteur d'eau augmente. Nous avons fait varier le nombre de particules (résolution spatiale) afin d'étudier la convergence du schéma. L'augmentation de la densité en particules améliore les résultats et retarde le deuxième et le troisième pic ce pression. Nous savons que, dans le cas réel, une poche d'air est générée après le premier pic (voir premier image de la figure 6), qui peut influencer les deux pics suivants. Prochainement, nous allons donc prendre en compte une phase air pour en étudier l'influence.

En pratique, avec l'objectif d'étudier la survivabilité des dispositifs de récupération de l'énergie marine, nous nous intéressons principalement aux efforts exercées sur le mur. Nous avons intégré la pression sur la paroi (cf. figure 7), afin d'obtenir la force résultante :

$F=\left.\int_{0}^{\eta_{\max }} P(y) d y\right|_{x=L_{x}}$

La force maximale est obtenue quand le premier pic de pression survient. Sa valeur augmente avec le nombre de particules, se rapprochant progressivement d'une valeur maximale. 


\section{XIII ${ }^{\text {èmes }}$ Journées Nationales Génie Côtier - Génie Civil \\ Dunkerque, 2-4 juillet 2014}

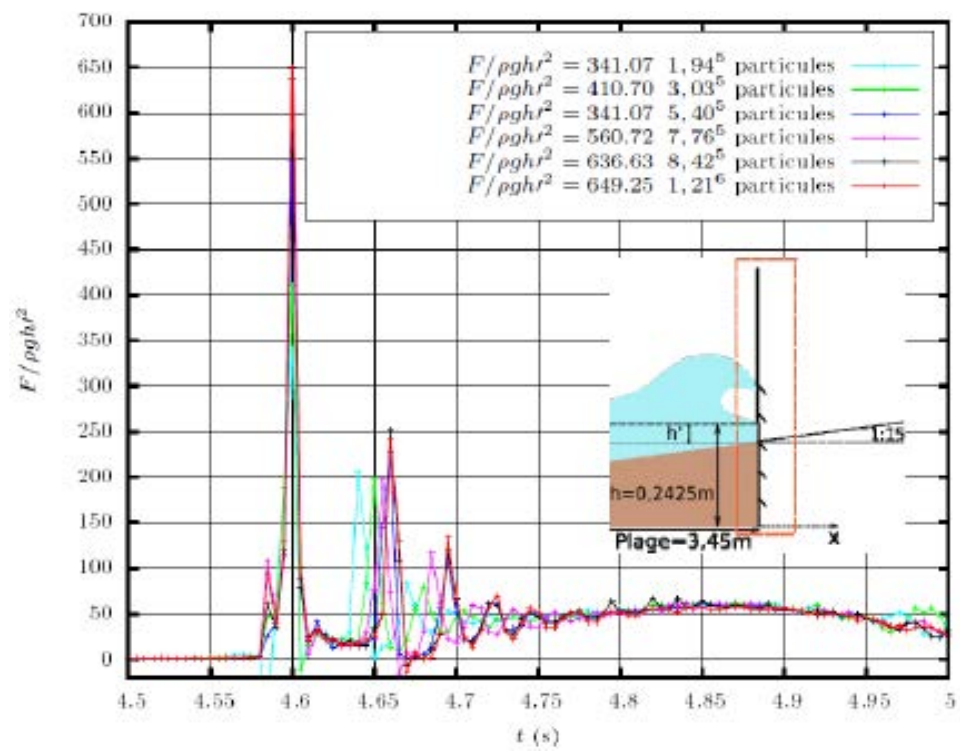

Figure 7. Évolution temporelle de la force exercée sur la paroi (équation 6) adimensionnée par $\rho g h^{\prime 2}$ avec $h^{\prime}$ la hauteur d'eau au point d'impact (figure 8).

\section{4. Étude d'un cas académique : impact du jet triangulaire}

Dans le but de comparer notre code JOSEPHINE et le code THETIS (MOKRANI et al., 2012) sur le problème d'impact, nous avons commencé par un cas-test idéalisé où la surface du jet est rectiligne. Une étude théorique de (WU, 2007) établit le lien entre l'inclinaison locale de l'interface défini par l'angle $\alpha$ et la dynamique de l'impact. En effet, ce cas d'étude est une simplification du problème d'impact de la vague (voir figure 8 , à gauche).

Les schémas de la figure 8 à droite présentent l'écoulement simulé. Le jet triangulaire, qui forme un angle $\alpha$ par rapport à l'axe horizontal, se déplace à une vitesse constante $u_{0}$ selon le même axe. Nous nous focalisons sur la reproduction et l'estimation de ce profil de pression sur la paroi verticale en un instant donné.

Pour ce problème, l'impact est assez violent avec un angle $\alpha$ important. Afin d'améliorer les résultats, nous avons ajouté une viscosité artificielle dans l'équation de quantité de mouvement. L'incrustation de la figure 9 montre la représentation de particules en $t=0,2 \mathrm{~s}$ pour $\alpha=45^{\circ}$, avec la viscosité artificielle. Nous constatons que la trajectoire des particules le long du jet vertical, ainsi que la répartition de la pression sur la paroi (cf. figure 9), sont ici nettement améliorées. En faisant varier le pas de temps, les résultats convergent et sont en bon accord avec la méthode VOF (MOKRANI et al., 2012), ainsi qu'avec le modèle analytique (WU, 2007). Cette viscosité artificielle améliore également la stabilité du schéma, nous permettant ainsi d'augmenter le pas de temps de simulation et de réduire les temps de calcul. 

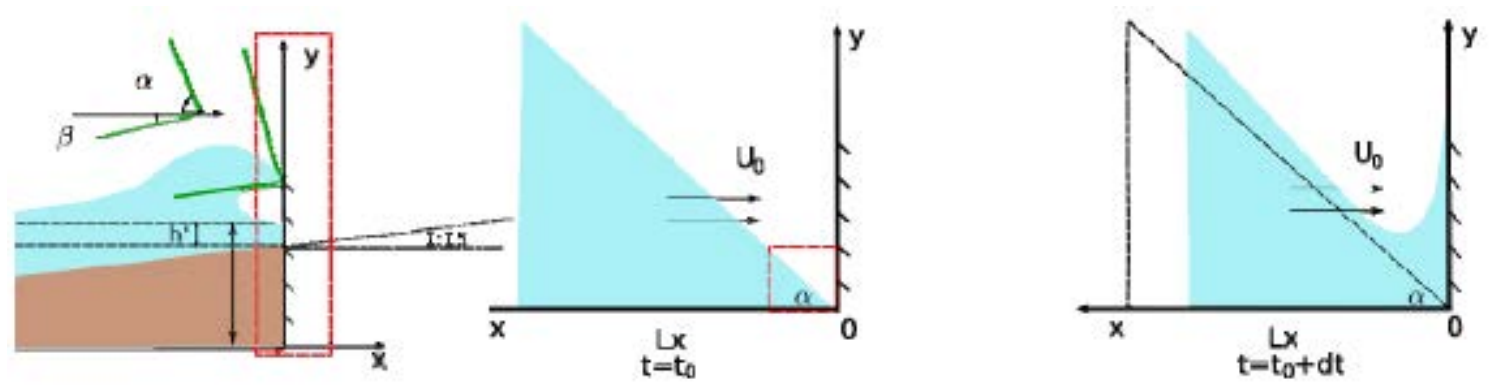

Figure 8. Schéma de cas d'impact d'un jet triangulaire inspiré de (MOKRANI, 2012).
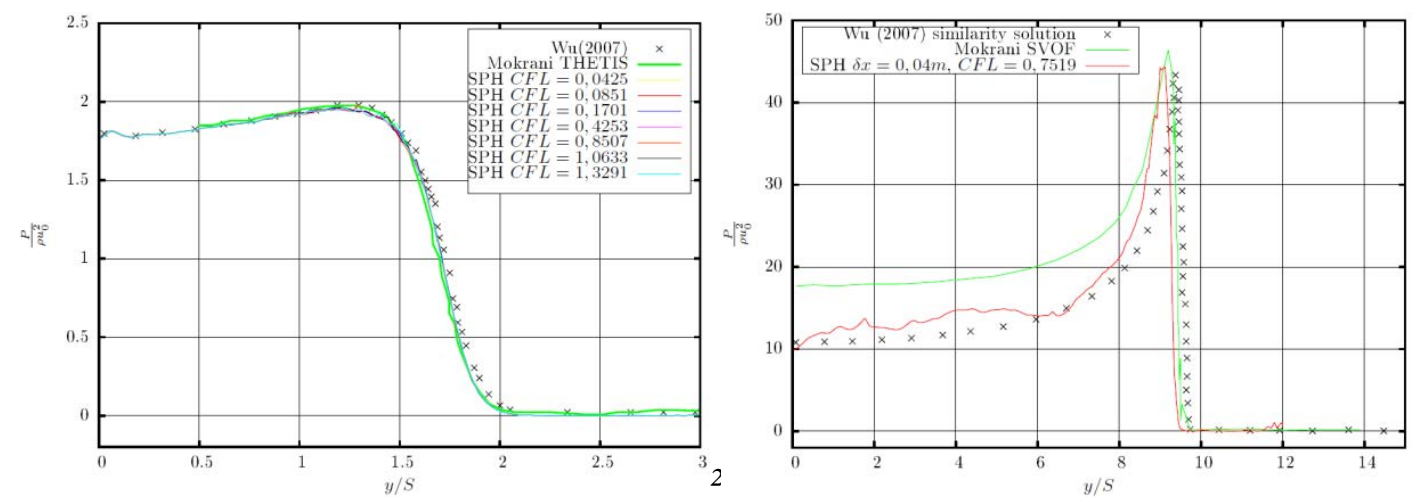

Figure 9. Gauche, $\alpha=45^{\circ}$, répartition de la pression sur la paroi en $t_{o b s}=0,2 \mathrm{~s}$, $\delta x=0,0141 \mathrm{~m}$ et Droite, $\alpha=80^{\circ}$, répartition de la pression sur la paroi en $t_{o b s}=0,2 \mathrm{~s}$. La position sur le mur est adimensionnée par $S=U_{0} t_{o b s}$.

Dans l'article de (MOKRANI et al., 2012), pour $\alpha>60^{\circ}$, les calculs Navier-Stokes VOF ne respectent plus l'autosimilarité des solutions de (WU, 2007). Nous avons essayé d'augmenter l'angle $\alpha$, jusqu'à $80^{\circ}$. La figure 9 montre le résultat avec le pas d'espace $\delta x=0,04 m$ et un $C F L=0,7519$. Il est plus proche du résultat analytique de (WU, 2007) que ceux de THETIS. La méthode SPH est généralement bien adaptée aux problèmes d'impacts violents. Nous allons raffiner le maillage à l'instant initial et diminuer le pas de temps de simulation pour améliorer le résultat.

\section{Conclusions et perspectives}

Nous avons bien généré et propagé un soliton dans un canal à houle numérique. Les impacts de soliton, les pics de pression et les efforts sur le mur ont été étudiés. La capacité de la méthode SPH à résoudre des problèmes d'impacts a été prouvée en traitant un cas académique de "jet triangulaire".

Nous allons prochainement appliquer la variante diphasique du code de calcul afin d'observer le rôle de la phase air sur le cas "air pocket impact". Nos résultats seront comparés avec ceux fournis par le code THETIS et les expériences sur les différents types d'impact. 


\section{XIII ${ }^{\text {èmes }}$ Journées Nationales Génie Côtier - Génie Civil \\ Dunkerque, 2-4 juillet 2014}

\section{Références bibliographiques}

BOUSSINESQ J. (1872). Théorie des ondes et des remous qui se propagent le long d'un canal rectangulaire horizontal, en communiquant au liquide contenu dans ce canal des vitesses sensiblement pareilles de la surface au fond. J. Math. Pures Appl., Vol. 17(2), pp 55-108.

COLAGROSSI A., LANDRINI M. (2003). Numerical simulation of interfacial flows by smoothed particle hydrodynamics. Journal of Computational Physics, Vol. 191, pp 448-475. http://dx.doi.org/10.1016/S0021-9991(03)00324-3

CHERFILS J.M., PINON G., RIVOALEN E. (2012). JOSEPHINE: A parallel SPH code for free-surface flows. Computer Physics Communications, Vol. 183, pp 1468-1480. http://dx.doi.org/10.1016/j.cpc.2012.02.007

FERRARI A., DUMBSER M., TORO E.F., ARMANINI A. (2009). A new 3d parallel sph scheme for free surface flows. Comput. Fluids, Vol. 38, 1203-1217. http://dx.doi.org/10.1016/j.compfluid.2008.11.012

GORING D.G. (1978). Tsunamis, the propagation of long waves onto a shelf. $\mathrm{PhD}$ thesis, California Institute of Technology Pasadena, California.

GUILCHER P., OGER G., BROSSET L., JACQUIN E., GRENIER N., LE TOUZÉ D. (2010). Simulation of liquid impacts with a two-phase parallel SPH model. Proceedings of $20^{\text {th }}$ International Offshore and Polar Engineering Conference, June 20-26, Bejing, China.

HOFLAND B., KAMINSKI M., WOLTERS G. (2011). Large scale wave impacts on a vertical wall. Coastal Engineering Proceedings, 1(32). structures.15. http://dx.doi.org/10.9753/icce.v32.structures.15

KIMMOUN O., SCOLAN Y.M., MRAVAK Z. (2008). Communication privée. Minislo - séminaire clarom océano-météo et hydrodynamique 2008.

MOKRANI C. (2012). Impacts de vagues déferlantes sur un obstacle vertical. Modèle théorique et estimation numérique des pics de pression. Thèse Université de Pau et des Pays de l'Adour.

MOKRANI C., ABADIE S., ZIBOUCHE K. (2012). Lien entre la forme locale de la surface libre et les pressions d'impact générées par une vague déferlante sur un ouvrage. Journées Nationales Génie Côtier - Génie Civil, Cherbourg. pp 109-116. http://dx.doi.org/10.5150/jngcgc.2012.012-M

RAFIEE A., DUTYKH D., DIAS F. (2013). Numerical simulation of wave impact on a rigid wall using a two--phase compressible SPH method. ArXiv e-prints. http://arxiv.org/abs/1306.0769v1

WU G. (2007). Fluid impact on a solid boundary. Journal of Fluids and Structures, Vol. 23, pp 755-765. http://dx.doi.org/10.1016/j.jfluidstructs.2006.11.002 
Thème 1 - Hydrodynamique côtière 